\title{
PHOTOMETRIC CLASSIFICATION OF B-TYPE STARS
}

\author{
D. L. CRAWFORD
}

Kitt Peak National Observatory, Tucson, Ariz., U.S.A.

\begin{abstract}
Several photometric techniques for classification of B-type stars exist. This note describes another one, now nearly ready for publication.
\end{abstract}

We have observed most of the stars with spectral types B5 and earlier, and brighter than $m_{v}=6.5$ with the $u v b y$ and $\mathrm{H} \beta$ systems. In addition, we have data for the following clusters or associations: $h$ and $\chi$ Per, NGC 6231, $\alpha$ Per, Pleiades, IC 2602, IC 2391, III Cep, and Sco-Cen. Good MK types exist for most of these stars, and $U B V$ data is available for many of them.

Figures 1 and 2 show the relations between $(b-y)$ and $(B-V)$ and between $(u-b)$ and $(U-B)$ for the $\mathrm{B}, \mathrm{A}$, and $\mathrm{F}$-type stars we use as standards. Table I gives the mean values of several MK spectral types. The unreddened values were determined as des-

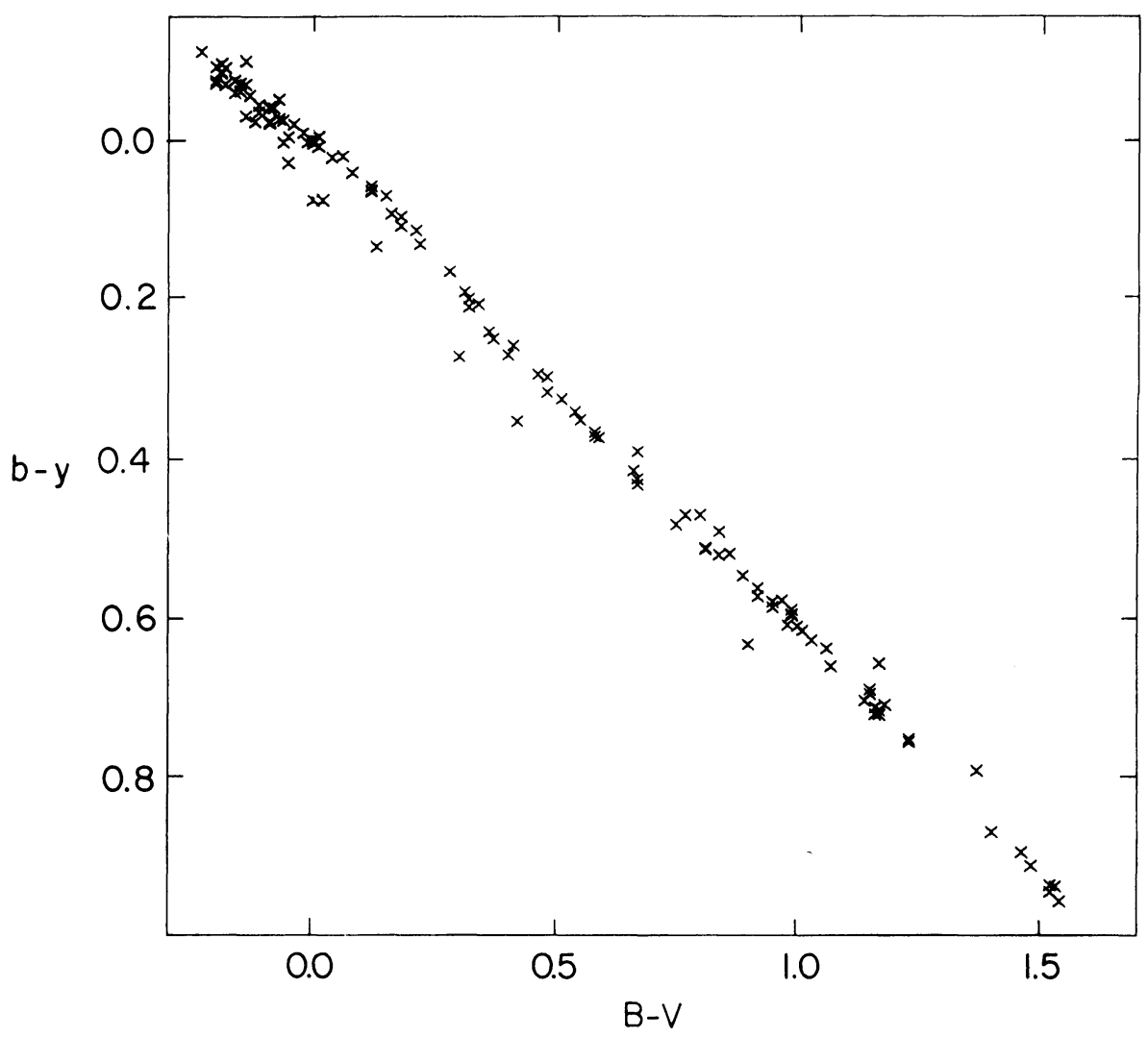

Fig. 1. 
PHOTOMETRIC CLASSIFICATION OF B-TYPE STARS

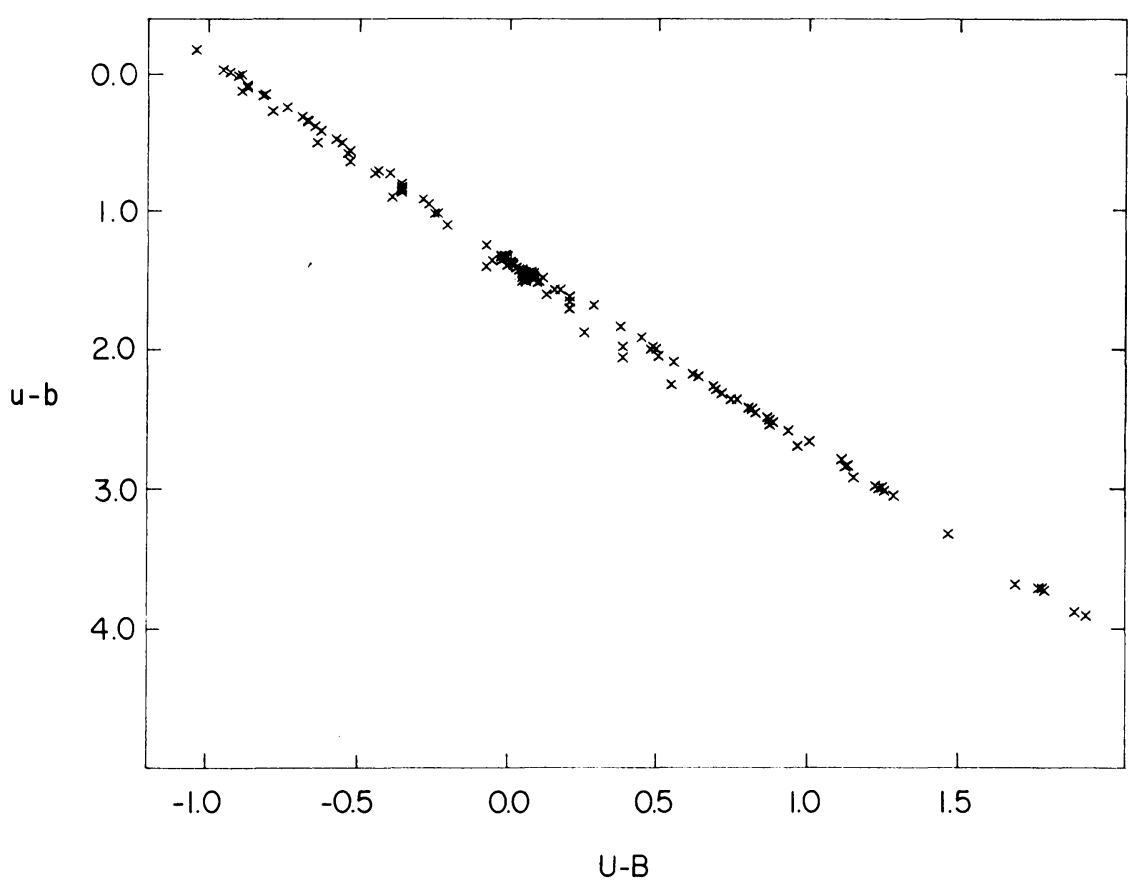

Fig. 2.

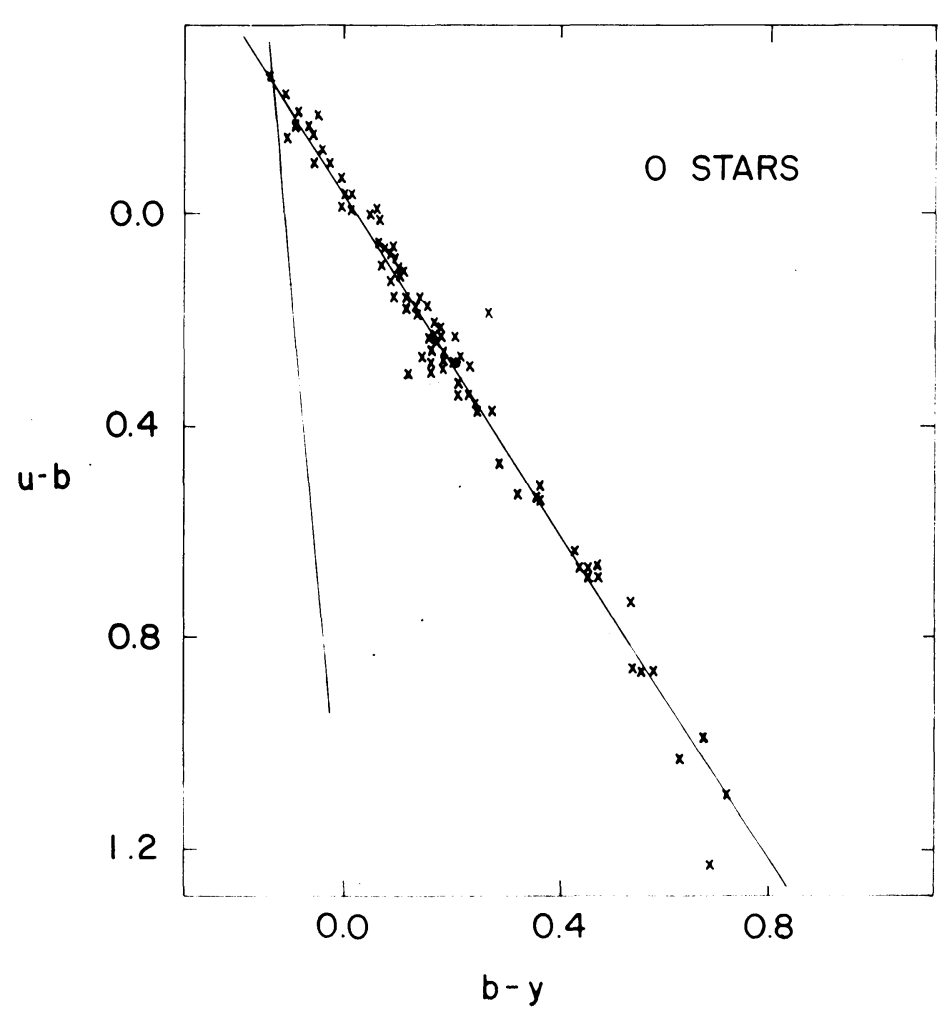

Fig. 3. 


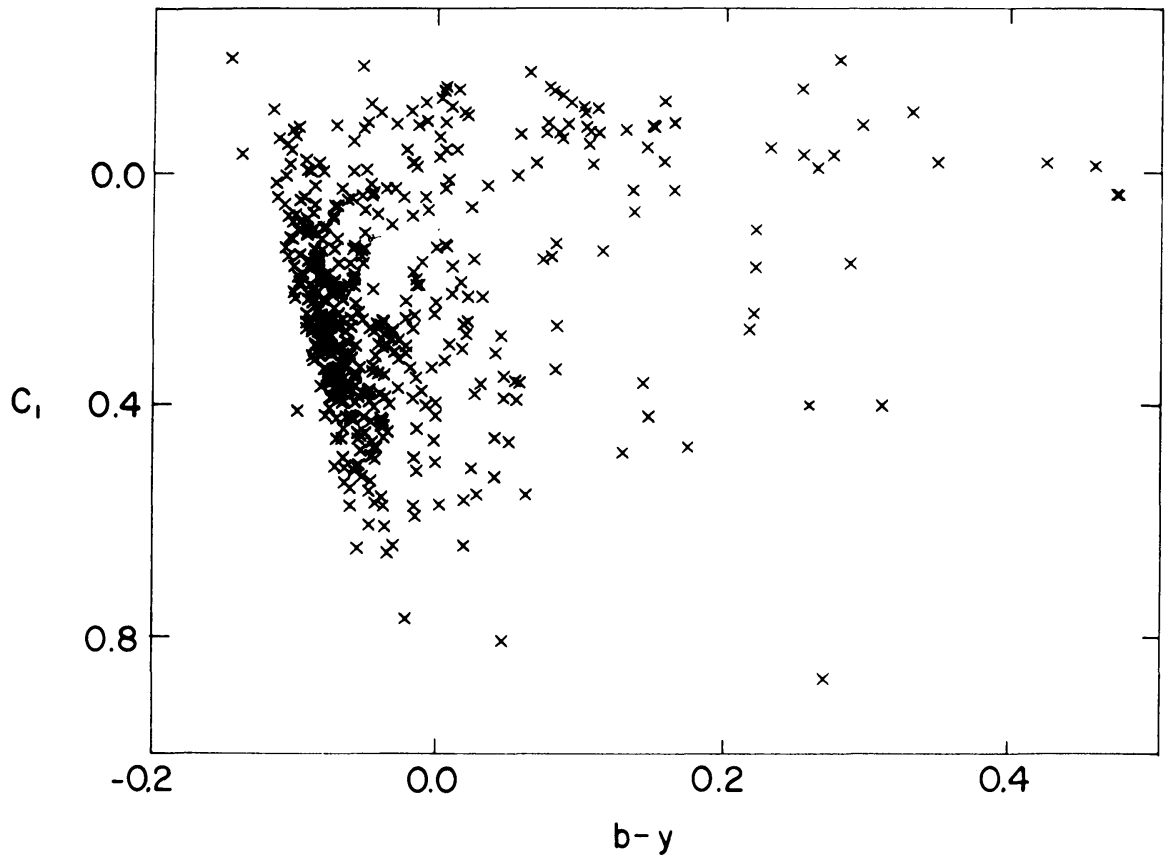

Fig. 4.

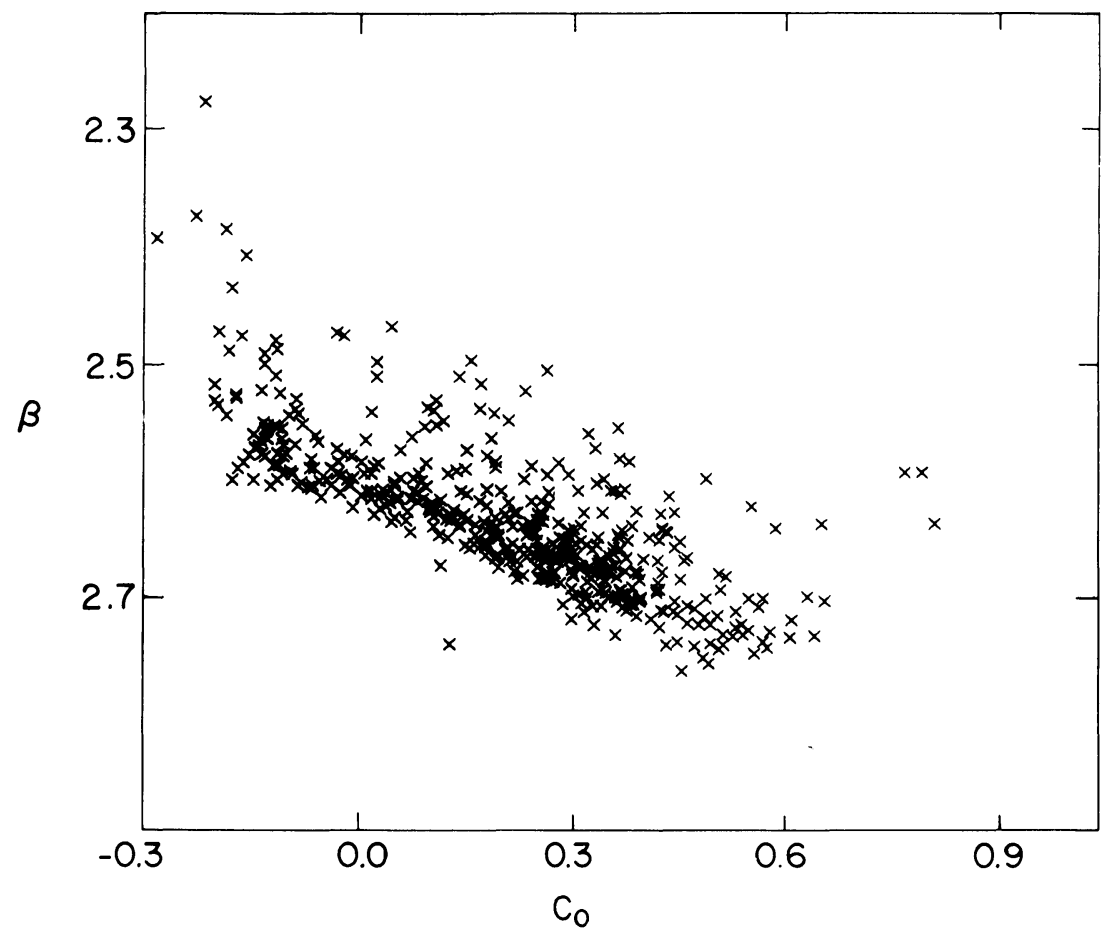

Fig. 5. 


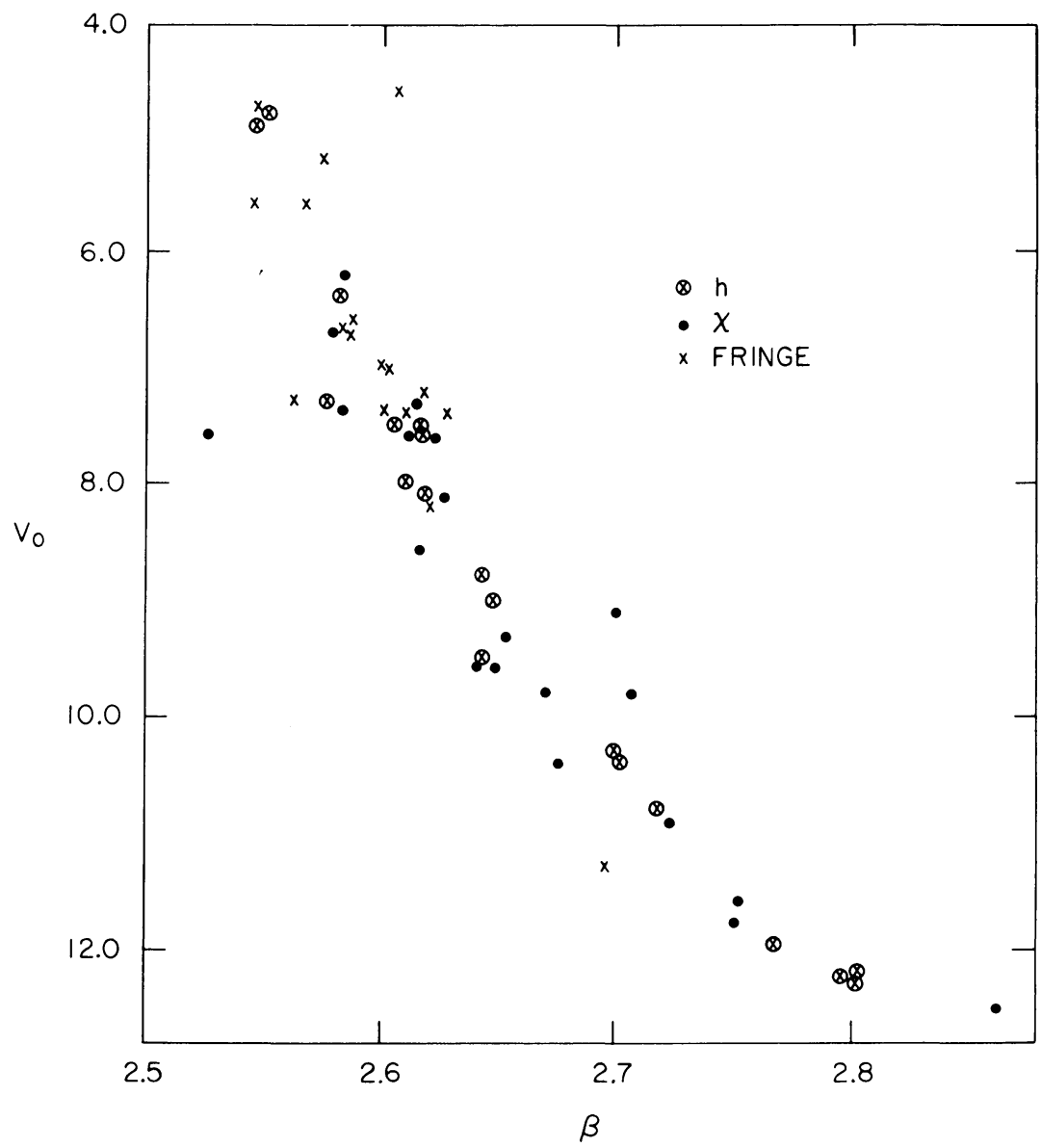

Fig. 6.

TABLE I

\begin{tabular}{lrrlll}
\hline MK type & \multicolumn{1}{c}{$C_{0}$} & $(b-y)_{0}$ & $\beta($ ZAMS) & $M_{v}$ & $(U-B)_{0}$ \\
\hline & & & & & \\
$\leqslant$ O8 & -0.15 & & $2.575:$ & -5.5 & -1.15 \\
O9 & -0.12 & -0.13 & 2.590 & -4.6 & -1.10 \\
B0 & -0.07 & -0.12 & 2.608 & -3.9 & -1.05 \\
B1 & 0.02 & -0.11 & 2.629 & -2.9 & -0.96 \\
B2 & 0.15 & -0.10 & 2.658 & -1.9 & -0.84 \\
B3 & 0.33 & -0.09 & 2.701 & -1.0 & $-0.67:$ \\
B4 & 0.37 & -0.08 & 2.709 & -0.8 & $-0.63:$ \\
B5 & 0.42 & -0.07 & 2.720 & -0.6 & -0.59
\end{tabular}




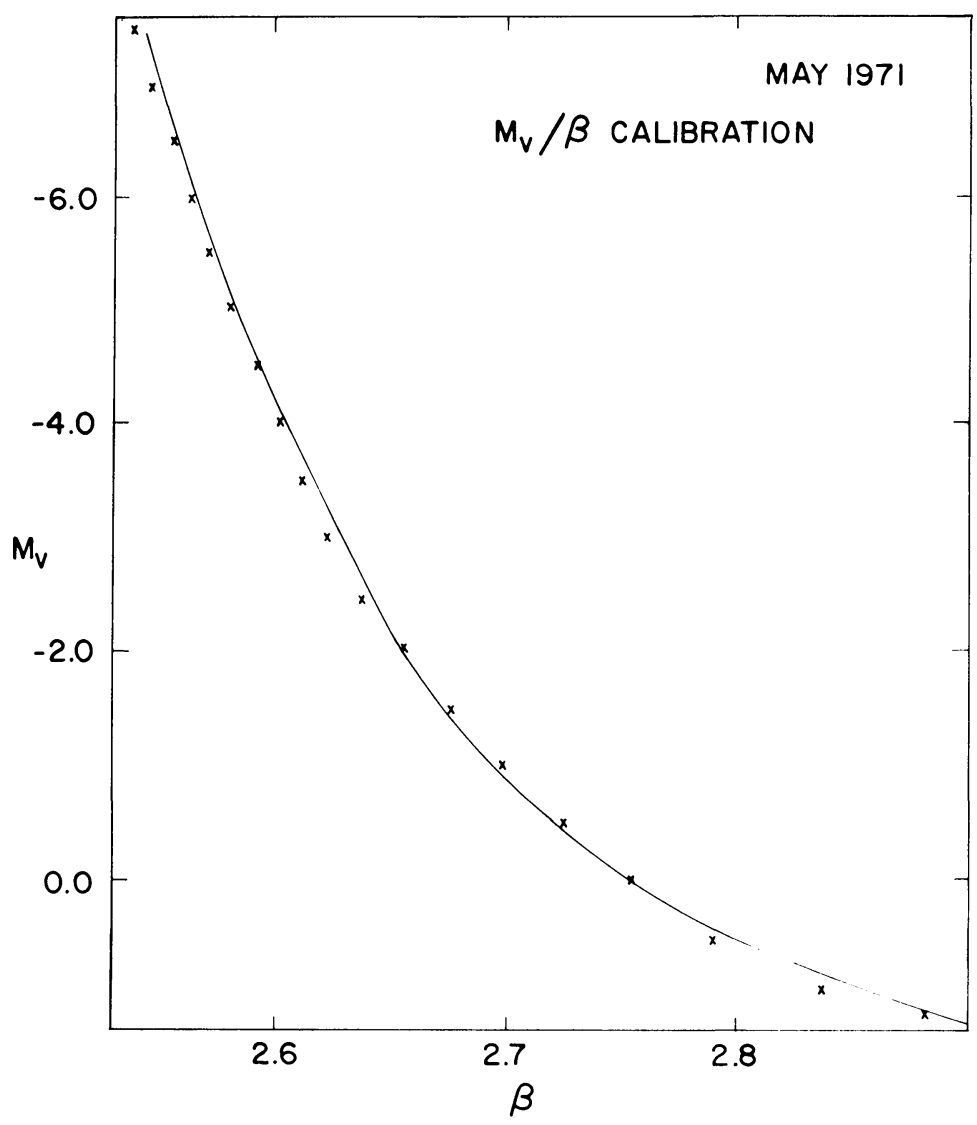

Fig. 7.

cribed by Crawford et al. (1970). Figure 3 shows the relations between $(u-b)$ and $(b-y)$ for a number of O-type stars.

The relation between the reddening lines for $(u-b) /(b-y)$ and the other indices are

$$
\begin{aligned}
E\left(c_{1}\right) & =0.2 E(b-y) \\
E\left(m_{1}\right) & =-0.3 E(b-y) \\
E(b-y) & =0.7 E(B-V) \\
E(u-b) & =1.7 E(b-y) .
\end{aligned}
$$

Figure 4 shows the relation between $c_{1}$ and $b-y$ for the bright B-type stars. The rather sharp left envelope can be taken as the preliminary intrinsic color relation: $(b-y)_{0}$ in terms of $c_{1}$. Figure 5 shows the relation between $\beta$ and $c_{1}$ for the bright B-type stars. The $\beta$ parameter is primarily a measure of luminosity and the $c_{1}$ parameter a measure of temperature. The lower envelope is the zero age line; data for stars of the youngest clusters lie nearly along this envelope. Comparison of the location of the data points in this diagram with the star's MK type indicates a very good relation 
between the two systems, though there are certainly some deviating stars. Effects due to emission of $\mathrm{H} \beta$ or $\mathrm{V} \sin i$ effects apparently do not cause serious problems.

Time, nor the conference subject, do not permit a discussion of calibration problems here, but Figure 6 shows the relations between $\beta$ and $V_{0}$ for the stars of the $h$ and $\chi$ Per group. Similar relations exist for other groups, and a fitting of such individual relations leads to a calibration of $M_{v}$ in terms of $\beta$ (see Figure 7) - the second dimension for the photometric classification: the $c_{0}$ parameter being the first dimension.

Details of the classification and the calibrations will be published shortly in the Astronomical Journal.

\section{Reference}

Crawford, D. L., Glaspey, J. W., and Perry, C. L.: 1970, Astron. J. 75, 822. 